\title{
Deep Laser Cooling and Efficient Magnetic Compression of Molecules
}

\author{
L. Caldwell, J. A. Devlin, ${ }^{\dagger}$ H. J. Williams, N. J. Fitch, E. A. Hinds, B. E. Sauer, and M. R. Tarbutt \\ Centre for Cold Matter, Blackett Laboratory, Imperial College London, \\ Prince Consort Road, London SW7 2AZ, United Kingdom
}

(Received 19 December 2018; revised manuscript received 27 February 2019; published 16 July 2019)

\begin{abstract}
We introduce a scheme for deep laser cooling of molecules based on robust dark states at zero velocity. By simulating this scheme, we show it to be a widely applicable method that can reach the recoil limit or below. We demonstrate and characterize the method experimentally, reaching a temperature of 5.4(7) $\mu \mathrm{K}$. We solve a general problem of measuring low temperatures for large clouds by rotating the phase-space distribution and then directly imaging the complete velocity distribution. Using the same phase-space rotation method, we rapidly compress the cloud. Applying the cooling method a second time, we compress both the position and velocity distributions.
\end{abstract}

DOI: 10.1103/PhysRevLett.123.033202

There has been rapid progress in laser cooling of molecules in recent years. Several species have been cooled [1-6], and 3D magneto-optical traps (MOTs) have been demonstrated for a few [7-11]. Sub-Doppler cooling [8,12] and internal state control $[13,14]$ have been developed, and the molecules stored in magnetic and optical traps $[13,15,16]$. These laser-cooled molecules can be used to test fundamental physics [17,18], simulate many-body quantum systems [19], process quantum information [20-22], and study quantum chemistry [23]. All these applications require, or would benefit from, lower temperatures and higher densities. Here, we address both requirements, demonstrating a method that cools $\mathrm{CaF}$ molecules to $5 \mu \mathrm{K}$, and a method that efficiently compresses the cloud. We show that these two techniques can work together to increase the density and reduce the temperature. Finally, we present a technique that directly measures the complete velocity distribution of the ultracold sample. This thermometry is superior to the standard ballistic expansion method when the distribution is nonthermal, as happens, for example, with deep cooling schemes [24], velocity selection schemes [25], narrow-line MOTs [26] and ultracold plasmas [27]. It is also superior whenever distributions are cold but large, as happens in the present work and other applications including atom interferometry [28] and narrow-line MOTs [26].

First, we explain our new laser-cooling method. Figure 1(a) shows the hyperfine structure of the $A^{2} \Pi_{1 / 2} \leftarrow X^{2} \Sigma^{+}$ laser cooling transition in $\mathrm{CaF}$ at $606.3 \mathrm{~nm}$. Further details of the level structure are given in the Supplemental Material [29]. To make a MOT [8-10], radio-frequency sidebands are applied to the cooling light to address each of the ground state hyperfine components. The hyperfine structure of the excited state is not resolved. When the magnetic field is off and all sidebands are blue detuned, sub-Doppler cooling is effective and the molecules cool to $55 \mu \mathrm{K}[8,15,16]$.
This multifrequency molasses is shown as scheme (I) in Fig. 1(a). The molasses temperature is limited by the momentum diffusion arising from photon scattering. A good way to reduce this is to engineer a robust dark state-an eigenstate of the Hamiltonian that is not coupled by the light to any excited state-for molecules at zero velocity. This velocity selective coherent population trapping turns off the heating for the slowest particles, and has been used to cool atoms below the recoil limit [24,30]. Sometimes, the dark state is produced using a two-photon resonance between two hyperfine states. This method, often called $\Lambda$-enhanced gray molasses [31], was recently used by Cheuk et al. [12] to cool $\mathrm{CaF}$ to $5 \mu \mathrm{K}$. As shown by scheme (II) of Fig. 1(a), they turned off two sidebands and tuned the remaining two into resonance with the Raman transition between $F=1^{-}$and $F=2$ to engineer dark states that are superpositions of these levels. Nevertheless, a significant scattering rate remained, corresponding to an excited-state fraction of $1.3(2) \times 10^{-3}$. This is because the dark states are destabilized by off-resonant excitation (the frequency and polarization component targeting one hyperfine state may also excite the other), and by the $F=0$ level in the excited state, which couples to $F=1^{-}$but not to $F=2$. This suggests that even lower temperatures might be reached by finding more robust velocity-selective dark states, while retaining a strong cooling force. Our approach to achieve this is to use a single frequency component, blue detuned from all hyperfine levels, as illustrated by scheme (III) in Fig. 1(a). For stationary molecules, and for any polarization, there are two dark states that are superpositions of $F=2$ Zeeman sublevels. One is an eigenstate of the full Hamiltonian, including the kinetic energy operator. Moving molecules spend some of their time in bright states, where the average light shift can be large because the light has high intensity and is not too far detuned from $F=1^{-}$. Consequently, there can be a strong cooling force, but little scattering, for molecules at low 
(a)

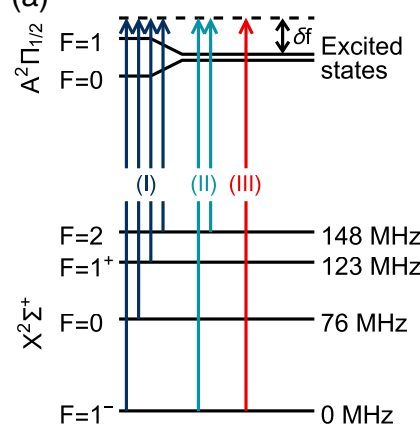

(b)

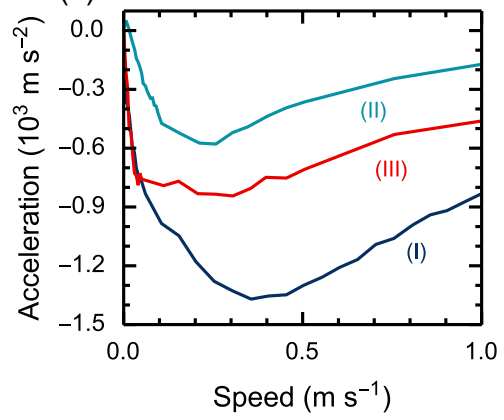

(c)

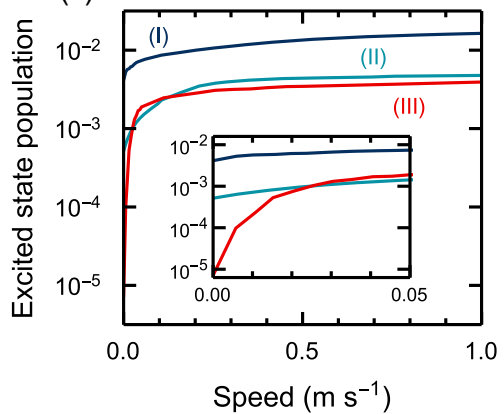

FIG. 1. (a) Hyperfine components of the laser cooling transition in CaF, with three cooling schemes shown: (I) multifrequency; (II) $\Lambda$ enhanced; (III) single frequency. (b) Steady-state acceleration vs speed and (c) excited-state fraction vs speed, obtained from optical Bloch equation simulations of each scheme. Simulation parameters are (I) total intensity $I=117 \mathrm{~mW} / \mathrm{cm}^{2}$, detuning $\delta f=20 \mathrm{MHz}$; (II) $I=50 \mathrm{~mW} / \mathrm{cm}^{2}, \delta f=30 \mathrm{MHz}$; (III) $I=340 \mathrm{~mW} / \mathrm{cm}^{2}, \delta f=8.3 \mathrm{MHz}$. Parameters for (I) and (II) are close to those that give the lowest measured temperatures $[8,12]$.

speeds. These are the requirements for efficient 3D cooling to the recoil limit and below [24,32].

To explore these ideas, we simulate all three schemes illustrated in Fig. 1(a). We use 3D optical Bloch equation simulations that include all relevant molecular levels and laser frequency components, and all six beams of the molasses $[33,34]$. The motion of the molecules is treated classically. Results of these simulations are shown in Figs. 1(b) and 1(c). Figure 1(b) shows that while scheme (I) gives the largest force over the widest range of speeds, scheme (III) provides just as high a damping constant at low speed. Figure 1(c) shows that, at all speeds, schemes (II) and (III) have lower excited-state population than scheme (I), and that in scheme (III) this drops to very low values at the lowest speeds, because the population is pumped into stable dark states at zero velocity [35]. This opens the possibility of cooling below the recoil limit, which would not be possible with the other schemes where a substantial scattering rate remains even at zero speed. Further discussion of the dark states involved in (II) and (III) is given in the Supplemental Material [29]. Using the data in Figs. 1(b) and 1(c), and the Fokker-PlanckKramers equation [34], we estimate a lower temperature limit, $T_{\text {low }}$, for each scheme. For (I), we predict $T_{\text {low }}=$ $11(1) \mu \mathrm{K}$, about 4 times lower than measured. The discrepancy arises because our method neglects heating due to fluctuations of the dipole force [34]. For (II), we predict $T_{\text {low }}=5.4(8) \mu \mathrm{K}$, consistent with measurements [12]. For (III), the predicted temperature is below the recoil limit of $0.44 \mu \mathrm{K}$. In this regime a full quantum mechanical treatment of the motion is needed.

Our experiments begin with a cloud of $2 \times 10^{4} \mathrm{CaF}$ molecules cooled to $\sim 55 \mu \mathrm{K}$ by scheme (I) $[8,9]$. To implement scheme (III), we switch off the cooling light, turn off the modulators that add sidebands to the laser, step the laser frequency so that the detuning from $F=1^{-}$is $\delta f$, and then after a settling period, turn the light back on with intensity $I$. The period between one molasses and the other is $700 \mu \mathrm{s}$. The repump lasers remain on at full intensity throughout. After holding the molecules in the single-frequency molasses for time $t_{\mathrm{sfm}}$, we measure the temperature, $T$.

We first measured $T$ using the standard ballistic expansion method. Using optimized molasses parameters (see later), we measure axial and radial temperatures of 8(1) and 6.7(6) $\mu \mathrm{K}$ by this method, where we have given statistical uncertainties only. At these low temperatures, the cloud expands by less than $1 \mathrm{~mm}$ in the time taken to leave the field of view, so the size is always dominated by the initial size $\left(\sigma_{0}>1 \mathrm{~mm}\right)$, and the velocity distribution is never clearly revealed. In this situation, small deviations from a Gaussian spatial distribution, and imaging aberrations near the edges of the field of view, can lead to systematic shifts that dominate the temperature measurements. Indeed, in the axial direction $(z)$, our data do not fit perfectly to the ballistic expansion model (see Supplemental Material [29]). The expansion time could be increased by magnetically levitating the cloud, but this introduces large systematic uncertainties due to the distribution of magnetic moments, even at large bias fields.

Because of these difficulties, we use the method illustrated in Fig. 2 to measure the axial temperature. After cooling, we turn off the light and apply a magnetic field, $B$, which has an offset $B_{0} \approx 80 \mathrm{G}$, a gradient along $z$ that cancels gravity for molecules with magnetic moment $\mu=\mu_{\mathrm{B}}$, and a curvature along $z$ that traps these molecules with an angular oscillation frequency $\omega$. The method for making this field is described in the Supplemental Material [29]. Our simulations predict that, after cooling, the molecules are uniformly distributed amongst the $m_{F}$ levels of $F=2$, with no population in other states. Figure 2(b) shows $\mu$ vs $B$ for these states, and the corresponding Zeeman shifts are shown in the Supplemental Material [29]. At $B \approx B_{0}$, molecules in $m_{F}=-2$ have $\mu=-\mu_{B}$ and are ejected, while all others have $\mu \approx \mu_{\mathrm{B}}$ and are confined. As illustrated in Fig. 2(c), their phase-space distribution 
(a)

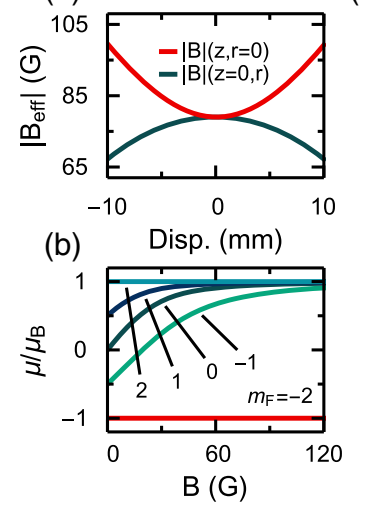

(c)

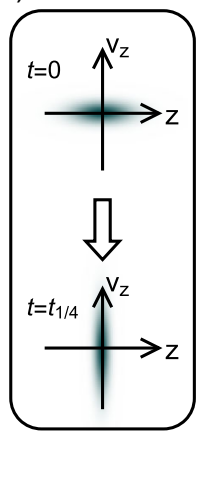

(d)

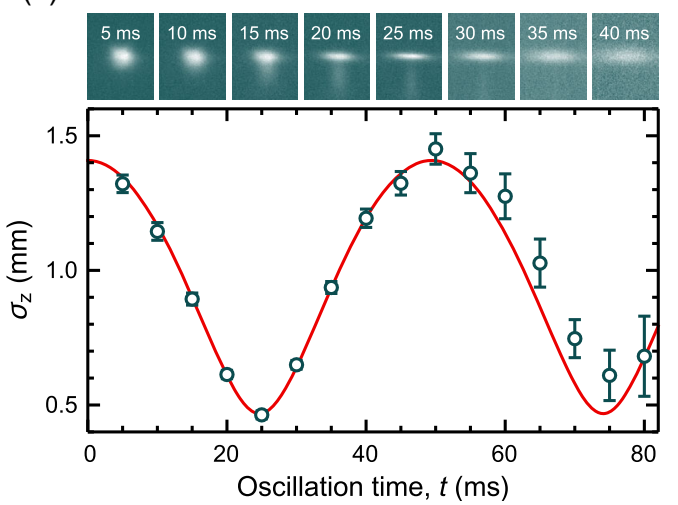

FIG. 2. Phase space rotation in a 1D magnetic trap. (a) Magnitude of effective magnetic field, $\left|B_{\text {eff }}\right|=|B|+m g z / \mu_{B}$, vs axial and radial displacements. Weak-field seeking molecules are trapped axially. (b) Magnetic moments $\mu$ of Zeeman sublevels in the $X^{2} \Sigma^{+}(N=$ $1, F=2)$ state, vs $B$. At large $B, \mu \approx \mu_{B}$ for $m_{F} \in\{-1,0,1,2\}$. (c) Phase space evolution in harmonic trap. After a quarter period, the position distribution is proportional to the initial velocity distribution. (d) Top: fluorescence images of molecules at $5 \mathrm{~ms}$ intervals, averaged over 50 shots; $z$ is vertical, and the field of view is $13.5 \mathrm{~mm}$ wide. Bottom: rms width in the $z$ direction vs time in trap, $\sigma_{z}(t)$. Error bars include systematic uncertainties. Line is a fit to Eq. (1).

rotates in the harmonic trap, so by imaging the cloud after a quarter of a period, we measure the initial velocity distribution. This is true for any initial distribution in phase space. When the initial velocity distribution is thermal with temperature $T$, and the initial position distribution is Gaussian with rms width $\sigma_{0}$, the position distribution remains Gaussian at all times $t$, with an rms width of

$$
\sigma(t)=\sqrt{\sigma_{0}^{2} \cos ^{2} \omega t+\frac{k_{B} T}{m \omega^{2}} \sin ^{2} \omega t}
$$

where $m$ is the mass. To measure this distribution, the magnetic field is turned off, the cooling light (with sidebands) immediately turned back on at the detuning used for the MOT, and the fluorescence imaged onto a CCD camera for $750 \mu \mathrm{s}$.

The upper row of Fig. 2(d) shows images at various times $t$. As expected, $\sim 80 \%$ of the molecules are trapped axially and slowly stretched radially. The remaining molecules are squeezed radially and accelerated downwards. We determine the rms width in $z$ of the trapped cloud $\sigma_{z}$ by integrating the image along $r$ over a central region of width $w_{\text {cut }}=4.43 \mathrm{~mm}$, then fitting to the resulting distribution. Using this central region reduces the effects of imaging aberrations near the edges of the field of view. For $t \leq 30 \mathrm{~ms}$, when the ejected molecules are still visible, we fit to the sum of two Gaussians with the position and width of each as free parameters. For later times a single Gaussian is sufficient. The lower panel of Fig. 2(d) shows $\sigma_{z}$ vs $t$. The line is a fit to Eq. (1), giving $\omega /(2 \pi)=10.12(2) \mathrm{Hz}$, within $10 \%$ of the value expected from a simple model of the coils, and $T=5.6(6) \mu \mathrm{K}$. We see that Eq. (1) fits well, apart from at late times where the measured size is inflated, mainly by the effect of defocusing as the cloud expands radially out of the imaging plane. This effect is reproduced by numerical modeling, and has a negligible effect on our determinations of $\omega$ and $T$. The temperature is related to the minimum width, $\sigma_{z, \min }$, obtained at time $t_{1 / 4}=\pi /(2 \omega)$, by $k_{B} T=$ $m \omega^{2} \sigma_{z, \min }^{2}$. All subsequent temperature data are obtained from images taken at $t_{1 / 4}$ and integrated over $w_{\text {cut }} / 2$. The statistical uncertainty in a single measurement made this way is $\sim 0.1 \mu \mathrm{K}$, far smaller than when measured by ballistic expansion. Systematic shifts and uncertainties are discussed in the Supplemental Material [29], are accounted for in all data presented, and result in a correction of $-1.4(7) \mu \mathrm{K}$ for the coldest clouds.

Figure 3(a) shows $T$ vs $t_{\text {sfm }}$ along with a fit to an exponential decay giving a $1 / e$ time constant of 0.52 (6) ms. A similar thermalization time constant of 0.41 (7) $\mathrm{ms}$ is predicted by the simulations. Figure 3(b) shows how $T$ depends on $\delta f$. The temperature decreases as $\delta f$ is tuned from negative to positive, reaches its lowest values for $\delta f>180 \mathrm{MHz}$, and is insensitive to $\delta f$ in this region, making the cooling robust. Simulations show the same dependence of $T$ on $\delta f$, including the mysterious bump near $50 \mathrm{MHz}$, but at lower temperatures throughout. Figure 3(c) shows that $T$ is insensitive to $I$ at high intensity, but increases at lower intensities which we attribute to a longer damping time at low $I$. Figure 3(d) shows that $T$ varies quadratically with background magnetic field, with curvature $9(1) \times$ $10^{-4} \mu \mathrm{K} \mathrm{mG}^{-2}$. Simulations also show a quadratic dependence, but with a curvature 4 times higher. The lowest temperature is obtained when all three field components are zero (measured within $10 \mathrm{mG}$ ). After optimizing all parameters, we measure $T=5.4(7) \mu \mathrm{K}$. This is consistent (within $2 \sigma$ ) with the value measured by ballistic expansion, but more reliable for the reasons discussed above and in the Supplemental Material [29]. Our simulations suggest that considerably lower temperatures are feasible, so we speculate 

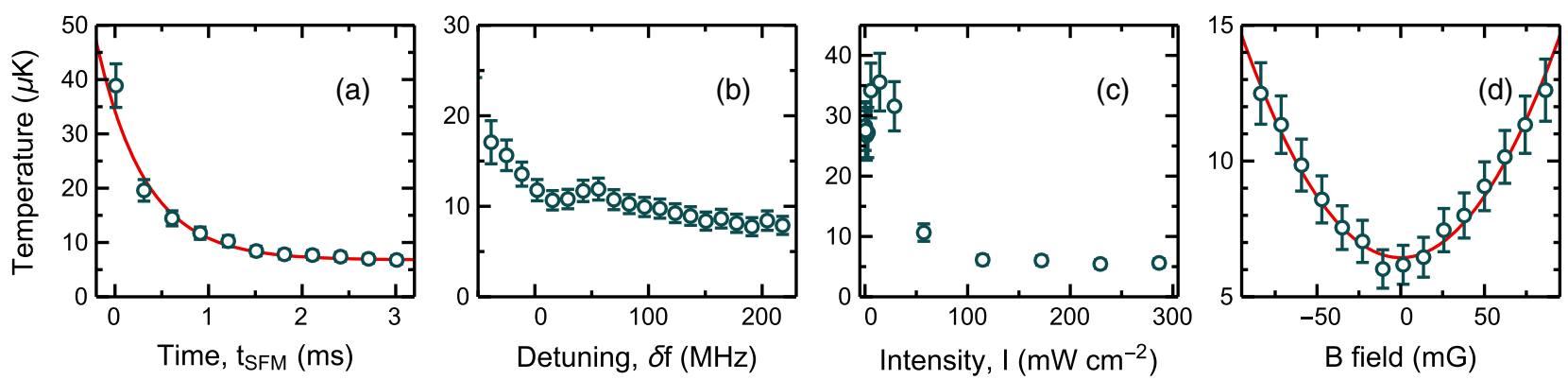

FIG. 3. Temperature vs single-frequency molasses parameters. (a) $T$ vs $t_{\mathrm{sfm}}$, when $I=287 \mathrm{~mW} \mathrm{~cm}^{-2}$ and $\delta f=164 \mathrm{MHz}$. Line: fit to $T=T_{0}+\left(T_{\mathrm{i}}-T_{0}\right) e^{-t_{\mathrm{sfm}} / \tau}$, giving $\tau=0.52(6) \mathrm{ms}$. (b) $T$ vs $\delta f$ when $t_{\mathrm{sfm}}=5 \mathrm{~ms}$ and $I=287 \mathrm{~mW} \mathrm{~cm}^{-2}$. (c) $T$ vs $I$ when $t_{\text {sfm }}=5 \mathrm{~ms}$ and $\delta f=164 \mathrm{MHz}$. (d) $T$ vs one component of $B$, after optimization of the other two components; $t_{\mathrm{sfm}}=5 \mathrm{~ms}, I=287 \mathrm{~mW} \mathrm{~cm}{ }^{-2}$ and $\delta f=164 \mathrm{MHz}$. Line: fit to $T=T_{0}+\alpha B^{2}$, giving $\alpha=9(1) \times 10^{-4} \mu \mathrm{K} \mathrm{mG}^{-2}$. Points give average and standard error of 50 experimental runs. Error bars are dominated by systematic uncertainties.

that the temperature reached here may be limited by a timevarying magnetic field or laser polarization, which could be improved. We observe no loss of molecules in the cooling step, other than the $20 \%$ lost when measuring $T$, and the data fit well to a single thermal distribution, so we conclude that all the molecules are cooled to the same low temperature.

As seen in Fig. 2(d), the cloud is compressed by a factor 3 at time $t_{1 / 4}$. It is common to compress magnetically trapped atoms [36], and recently molecules [15], by adiabatically increasing the trap frequency $\omega$ or field gradient $A$. Using this method, $\sigma$ scales only as $\omega^{-1 / 2}$ in a harmonic trap or as $A^{-1 / 3}$ in a quadrupole trap [36]. Thus, even modest reductions in cloud size require large field gradients, which must be maintained for long times to be adiabatic. Our rapid compression method is more effective because $\sigma$ scales as $\omega^{-1}$. The compression heats the cloud, but it can be recooled by applying the molasses a second time. This sequence increases the phase-space density provided (i) the cooling is fast enough that reexpansion during the second cooling phase is small, (ii) the velocities after compression are within the capture velocity of the molasses, and (iii) the magnetic field used for compression can be turned off rapidly enough that the molasses is effective. The first two conditions are easily satisfied, but the third is difficult. The $\sim 80 \mathrm{G}$ field must be rapidly reduced below $50 \mathrm{mG}$, but eddy currents can produce large, slowly decaying fields. We switch the coils off in a way that minimizes these effects, as detailed in the Supplemental Material [29], then reapply the singlefrequency molasses for $5 \mathrm{~ms}$, and finally measure $T$. Since the cloud is now much smaller, ballistic expansion measurements are reliable. Using this method, we measure $\sigma_{0}=$ $0.73(1) \mathrm{mm}$ and $T=14(1) \mu \mathrm{K}$, limited by the residual magnetic field, which could be reduced further. A longer wait between turning off the trap and re-applying the cooling reduces $T$ at the expense of $\sigma_{0}$. The complete cycle of cooling, compressing, and recooling reduces the axial size by a factor 2 , reduces the temperature by a factor 4 , and retains $80 \%$ of the molecules. A greater $\omega$ will give stronger compression, and the compression improves if $T$ is lowered further since $\sigma \propto T^{1 / 2}$.

In summary, we have demonstrated a simple, robust method that cools molecules to $5 \mu \mathrm{K}$. The method works by reducing the scattering rate to low values for the slowest molecules. Simulations suggest that temperatures down to the recoil limit of $0.44 \mu \mathrm{K}$, or even lower, should be possible, and we are studying how to achieve that. This cooling should work for all molecular species laser cooled so far, and the principles for engineering robust velocityselective dark states apply to new species too. We have developed a technique for directly measuring the velocity distribution of ultracold clouds that works for all phasespace distributions, and is superior to ballistic expansion for large clouds. Finally, we have shown that these ultracold clouds can be compressed using a conservative potential, and recooled after compression. For smaller clouds, the compression could be applied using an optical trap [16]. For example, a $50 \mathrm{~W}$ laser at $1 \mu \mathrm{m}$ with a waist of $300 \mu \mathrm{m}$ would, we estimate, compress the cloud to $\sim 90 \mu \mathrm{m}$. The compression demonstrated in one dimension can be extended by making a 3D harmonic potential, e.g., using a Ioffe-Pritchard trap. If the trap is isotropic, which is possible in this geometry [37], the cloud can be compressed in all directions. Large increases in the 3D phase space density can be achieved by these methods. This is important for applications in quantum simulation and information processing, studies of ultracold collisions, and cooling molecules towards quantum degeneracy.

Underlying data may be accessed from Zenodo [38] and used under the Creative Commons CCZero license.

We are grateful to Simon Cornish for valuable discussions that led to some of the methods used in this work. We thank J. Dyne and V. Gerulis for expert technical assistance and A. Guo for work on characterizing the imaging system. This work was supported by EPSRC under Grants No. EP/ M027716/1 and No. EP/P01058X/1. 
*m.tarbutt@imperial.ac.uk

${ }^{\dagger}$ Present address: CERN, 1211 Geneva, Switzerland.

[1] E. S. Shuman, J. F. Barry, and D. DeMille, Laser cooling of a diatomic molecule, Nature (London) 467, 820 (2010).

[2] M. T. Hummon, M. Yeo, B. K. Stuhl, A. L. Collopy, Y. Xia, and J. Ye, 2D Magneto-Optical Trapping of Diatomic Molecules, Phys. Rev. Lett. 110, 143001 (2013).

[3] V. Zhelyazkova, A. Cournol, T. E. Wall, A. Matsushima, J. J. Hudson, E. A. Hinds, M. R. Tarbutt, and B. E. Sauer, Laser cooling and slowing of CaF molecules, Phys. Rev. A 89, 053416 (2014).

[4] A. Prehn, M. Ibrügger, R. Glöckner, G. Rempe, and M. Zeppenfeld, Optoelectrical Cooling of Polar Molecules to Submillikelvin Temperatures, Phys. Rev. Lett. 116, 063005 (2016).

[5] I. Kozyryev, L. Baum, K. Matsuda, B. L. Augenbraun, L. Anderegg, A. P. Sedlack, and J. M. Doyle, Sisyphus Laser Cooling of a Polyatomic Molecule, Phys. Rev. Lett. 118, 173201 (2017).

[6] J. Lim, J. R. Almond, M. A. Trigatzis, J. A. Devlin, N. J. Fitch, B. E. Sauer, M. R. Tarbutt, and E. A. Hinds, Laser Cooled YbF Molecules for Measuring the Electron's Electric Dipole Moment, Phys. Rev. Lett. 120, 123201 (2018).

[7] J. F. Barry, D. J. McCarron, E. B. Norrgard, M. H. Steinecker, and D. DeMille, Magneto-optical trapping of a diatomic molecule, Nature (London) 512, 286 (2014).

[8] S. Truppe, H. J. Williams, M. Hambach, L. Caldwell, N. J. Fitch, E. A. Hinds, B. E. Sauer, and M. R. Tarbutt, Molecules cooled below the Doppler limit, Nat. Phys. 13, 1173 (2017).

[9] H. J. Williams, S. Truppe, M. Hambach, L. Caldwell, N. J. Fitch, E. A. Hinds, B. E. Sauer, and M. R. Tarbutt, Characteristics of a magneto-optical trap of molecules, New J. Phys. 19, 113035 (2017).

[10] L. Anderegg, B. L. Augenbraun, E. Chae, B. Hemmerling, N. R. Hutzler, A. Ravi, A. Collopy, J. Ye, W. Ketterle, and J. M. Doyle, Radio Frequency Magneto-Optical Trapping of CaF with High Density, Phys. Rev. Lett. 119, 103201 (2017).

[11] A. L. Collopy, S. Ding, Y. Wu, I. A. Finneran, L. Anderegg, B. L. Augenbraun, J. M. Doyle, and J. Ye, 3D MagnetoOptical Trap of Yttrium Monoxide, Phys. Rev. Lett. 121, 213201 (2018).

[12] L. W. Cheuk, L. Anderegg, B. L. Augenbraun, Y. Bao, S. Burchesky, W. Ketterle, and J. M. Doyle, $\Lambda$-Enhanced Imaging of Molecules in an Optical Trap, Phys. Rev. Lett. 121, 083201 (2018).

[13] H. J. Williams, L. Caldwell, N. J. Fitch, S. Truppe, J. Rodewald, E. A. Hinds, B. E. Sauer, and M. R. Tarbutt, Magnetic Trapping and Coherent Control of Laser-Cooled Molecules, Phys. Rev. Lett. 120, 163201 (2018).

[14] J. A. Blackmore, L. Caldwell, P. D. Gregory, E. M. Bridge, R. Sawant, J. Aldegunde, J. Mur-Petit, D. Jaksch, J. M. Hutson, B. E. Sauer, M. R. Tarbutt, and S. L. Cornish, Ultracold molecules for quantum simulation: Rotational coherences in $\mathrm{CaF}$ and RbCs, Quantum Sci. Technol. 4, 014010 (2018).

[15] D. J. McCarron, M. H. Steinecker, Y. Zhu, and D. DeMille, Magnetic Trapping of an Ultracold Gas of Polar Molecules, Phys. Rev. Lett. 121, 013202 (2018).
[16] L. Anderegg, B. L. Augenbraun, Y. Bao, S. Burchesky, L. W. Cheuk, W. Ketterle, and J. M. Doyle, Laser cooling of optically trapped molecules, Nat. Phys. 14, 890 (2018).

[17] M. S. Safronova, D. Budker, D. DeMille, D. F. Jackson Kimball, A. Derevianko, and C. W. Clark, Search for new physics with atoms and molecules, Rev. Mod. Phys. 90, 025008 (2018).

[18] D. DeMille, J. M. Doyle, and A. O. Sushkov, Probing the frontiers of particle physics with tabletop-scale experiments, Science 357, 990 (2017).

[19] A. Micheli, G. K. Brennen, and P. Zoller, A toolbox for latticespin models with polar molecules, Nat. Phys. 2, 341 (2006).

[20] D. DeMille, Quantum Computation with Trapped Polar Molecules, Phys. Rev. Lett. 88, 067901 (2002).

[21] S. F. Yelin, K. Kirby, and R. Côté, Schemes for robust quantum computation with polar molecules, Phys. Rev. A 74, 050301(R) (2006).

[22] A. André, D. DeMille, J. M. Doyle, M. D. Lukin, S. E. Maxwell, P. Rabl, R. J. Schoelkopf, and P. Zoller, A coherent all-electrical interface between polar molecules and mesoscopic superconducting resonators, Nat. Phys. 2, 636 (2006).

[23] R. V. Krems, Cold controlled chemistry, Phys. Chem. Chem. Phys. 10, 4079 (2008).

[24] J. Lawall, S. Kulin, B. Saubamea, N. Bigelow, M. Leduc, and C. Cohen-Tannoudji, Three-Dimensional Laser Cooling of Helium Beyond the Single-Photon Recoil Limit, Phys. Rev. Lett. 75, 4194 (1995).

[25] J. K. Fox, H. A. Kim, S. R. Mishra, S. H. Myrskog, A. M. Jofre, L. R. Segal, J. B. Kim, and A. M. Steinberg, Classical and quantum analysis of one-dimensional velocity selection for ultracold atoms, J. Opt. B 7, 240 (2005).

[26] J. Grünert and A. Hemmerich, Sub-doppler magneto-optical trap for calcium, Phys. Rev. A 65, 041401(R) (2002).

[27] T. C. Killian, Ultracold neutral plasmas, Science 316, 705 (2007).

[28] T. Kovachy, J. M. Hogan, A. Sugarbaker, S. M. Dickerson, C. A. Donnelly, C. Overstreet, and M. A. Kasevich, Matter Wave Lensing to Picokelvin Temperatures, Phys. Rev. Lett. 114, 143004 (2015).

[29] See Supplemental Material at http://link.aps.org/ supplemental/10.1103/PhysRevLett.123.033202 for further details of dark states, magnetic field control, and systematic uncertainties in temperature measurements.

[30] A. Aspect, E. Arimondo, R. Kaiser, N. Vansteenkiste, and C. Cohen-Tannoudji, Laser Cooling below the One-Photon Recoil by Velocity-Selective Coherent Population Trapping, Phys. Rev. Lett. 61, 826 (1988).

[31] A. T. Grier, I. Ferrier-Barbut, B. S. Rem, M. Delehaye, L. Khaykovich, F. Chevy, and C. Salomon, $\Lambda$-enhanced subDoppler cooling of lithium atoms in $D_{1}$ gray molasses, Phys. Rev. A 87, 063411 (2013).

[32] F. Papoff, F. Mauri, and E. Arimondo, Transient velocityselective coherent population trapping in one dimension, J. Opt. Soc. Am. B 9, 321 (1992).

[33] J. A. Devlin and M. R. Tarbutt, Three-dimensional Doppler, polarization-gradient, and magneto-optical forces for atoms and molecules with dark states, New J. Phys. 18, 123017 (2016).

[34] J. A. Devlin and M. R. Tarbutt, Laser cooling and magnetooptical trapping of molecules analyzed using optical Bloch 
equations and the Fokker-Planck-Kramers equation, Phys. Rev. A 98, 063415 (2018).

[35] In the simulations, the cooling time is $0.8 \mathrm{~ms}$. In schemes (I) and (II) the populations have reached their steady-state values in this time, whereas in (III) the excited-state fraction continues to fall for longer cooling times.

[36] W. Ketterle, D. S. Durfee, and D. M. Stamper-Kurn, Making, probing and understanding Bose-Einstein Condensates, in
Proceedings of the International School of Physics Enrico Fermi, Course CXL, edited by M. Inguscio, S. Stringari, and C. Wieman (IOS Press, Amsterdam, 1999), pp. 67-176.

[37] T. Bergeman, G. Erez, and H. J. Metcalf, Magnetostatic trapping fields for neutral atoms, Phys. Rev. A 35, 1535 (1987).

[38] https://doi.org/10.5281/zenodo.2564130. 